\title{
Increased orienting to unexpected action outcomes in schizophrenia
}

\section{Elena Núñez Castellar ${ }^{1}$, Femke Houtman ${ }^{1}$, Wim Gevers ${ }^{2}$, Manuel Morrens ${ }^{3,4}$, Sara Vermeylen ${ }^{3,5}$, Bernard Sabbe ${ }^{3,5}$ and Wim Notebaert ${ }^{1 *}$}

1 Department of Experimental Psychology, Ghent University, Ghent, Belgium

2 Unité de Recherché en Neurosciences Cognitives, Université Libre de Bruxelles, Brussels, Belgium

${ }^{3}$ Collaborative Antwerp Psychiatric Research Institute, University of Antwerp, Antwerp, Belgium

4 Psychiatric Centre "BroedersAlexianen," Boechout, Belgium

${ }^{5}$ Psychiatric Centre "St. Norbertushuis," Duffel, Belgium

Edited by:

Claudia Danielmeier, Radboud

University, Netherlands

Reviewed by:

Jan R. Wessel, University of

California, San Diego, USA

Frederik Van Der Veen, Erasmus

University, Netherlands

\section{*Correspondence.}

Wim Notebaert, Department of Experimental Psychology, Ghent University, 9000 Ghent, Belgium. e-mail:wim.notebaert@ugent.be
Although some recent research has indicated reduced performance monitoring in patients with schizophrenia, the literature on this topic shows some remarkable inconsistencies. While most studies suggest diminished error signals following error responses, some studies reported normal post-error slowing, while others reported reduced post-error slowing. Here we review these studies and highlight the most important discrepancies. Furthermore, we argue that overall error rates are a mostly neglected issue that can at least partly explain these discrepancies. It has been reported previously that post-error slowing depends on the error rates. Participants or patients that make more errors are likely to show decreased post-error slowing. Therefore, when a group of patients is compared to a group of controls, it is extremely important to match error rates. For this purpose, we developed a procedure where we matched individuals' error rates. In a task where subjects had to press a response key corresponding to one of four colors we manipulated the difficulty on an individual basis by varying the discriminability between the colors. Schizophrenic patients and a group of controls were tested with this procedure showing that differences in accuracy disappear. Interestingly, we can see that in patients, the color values that were needed to reach similar levels of accuracy correlate with the Positive and Negative Syndrome Scale (PANSS) scale, with higher PANSS requiring more color. Most important, we found that schizophrenic patients have increased rather than decreased post-error slowing when the inter-trial interval (ITI) is short. This result can be interpreted within the framework of the orienting account, as it has been demonstrated previously that schizophrenic patients show increased distractibility.

Keywords: post-error slowing, outcome expectancy, schizophrenia, orienting account

\section{INTRODUCTION}

Impairments in monitoring and regulation of self-generated thoughts and behavior are considered as a hallmark in schizophrenia (Frith and Done, 1989; Leudar et al., 1994; Mlakar et al., 1994; Johns et al., 2001). The idea that these impairments could be related to deficits in the ability to monitor error responses and to make subsequent adjustments of behavior (Frith and Done, 1989; Mlakar et al., 1994) has received support during the last years. Studies performed with functional neuroimaging and electrophysiological methods have confirmed the existence of functional abnormality in error processing in schizophrenia. Specifically, functional neuroimaging studies have shown reduced error-related activation in the anterior cingulate cortex (ACC), a brain area considered to play a critical role in performance monitoring (Carter et al., 2001; Laurens et al., 2003; Kerns et al., 2005; Polli et al., 2008). Also, in line with these findings, event-related potential (ERP) studies have demonstrated that the amplitude of the error-related negativity (ERN), an ERP component generated in the ACC and observed as a negative deflection maximal at $50-150 \mathrm{~ms}$ following an erroneous response, is also disturbed
(Kopp and Rist, 1999; Alain et al., 2002; Mathalon et al., 2002; Bates et al., 2004). Specifically, it has not only been shown that the ERN amplitude is smaller in patients with schizophrenia when compared to controls but also that the ERN on correct trials is abnormally large in patients when compared to healthy subjects (Kopp and Rist, 1999; Alain et al., 2002; Mathalon et al., 2002).

Although this evidence suggests a clear deficit in performance monitoring in schizophrenia, studies that investigated behavioral adjustments following errors do not allow for a clear-cut conclusion. For instance, deficits in immediate error corrections were described in early behavioral studies (Malenka et al., 1982, 1986) but most recent studies observed intact error corrections in patients (Kopp and Rist, 1994, 1999; Polli et al., 2006, 2008; but for an exception see Turken et al., 2003). Likewise, some studies investigating behavioral slowing after errors (post-error slowing) reported a reduction or absence of post-error slowing in patients (Carter et al., 2001; Alain et al., 2002; Kerns et al., 2005) while others have shown that schizophrenic patients just as healthy individuals show post-error slowing (Kopp and Rist, 1999; Mathalon et al., 2002; Laurens et al., 2003; Polli et al., 2006, 2008). 
Most interesting, studies in which the neural response and behavioral error-related adjustments have been simultaneously investigated, all have observed diminished error-related activity but intact post-error slowing in patients with schizophrenia (Kopp and Rist, 1999; Mathalon et al., 2002; Laurens et al., 2003; Polli et al., 2008). These findings might suggest that error monitoring mechanisms and the mechanisms involved in implementing subsequent adjustments in behavior dissociate.

The aim of the present study is to further investigate behavioral changes following errors in patients compared to control subjects, when controlling for some methodological issues that have been largely ignored by previous research. We will specifically focus on post-error slowing, which is a behavioral adaptation effect that has been broadly investigated in healthy subjects (e.g., Rabbitt, 1966; Laming, 1968, 1979; Rabbitt and Rodgers, 1977; Gehring et al., 1993; Hajcak et al., 2003; Hajcak and Simons, 2008)

Recently, it has been suggested that the post-error slowing is related to the relative infrequency of errors which causes attentional capture that in turn delays processing of the following stimulus (Notebaert et al., 2009; Núñez Castellar et al., 2010). This idea is based on both behavioral and electrophysiological evidence. Behaviorally, it has been demonstrated that when the expectancy of error and correct responses is manipulated, the post-error slowing depends on the frequency of errors; when errors are more frequent than correct responses post-correct slowing instead of post-error slowing can be observed. This association has been confirmed by means of ERPs when investigating the neurophysiological correlates of the post-error and post-correct slowing. The results have shown that the $\mathrm{P} 3$, a component that has been associated with attentional orienting to unexpected events (see for a review Friedman et al., 2001) is correlated with posterror slowing. This suggests that post-error slowing is driven by attentional mechanisms elicited by the unexpected nature of the error (Notebaert et al., 2009), although this interpretation is still debated considering that several studies have reported the ERN amplitude in a single trial basis to be a predictor of the post-error slowing (Gehring et al., 1993; Debener et al., 2005)

Interestingly, schizophrenic patients show an increased vulnerability to distraction by novel stimuli (Grillon et al., 1990; Braff, 1993) and research investigating this deficit has provided convergent evidence suggesting that the reorienting of processing resources to salient novel stimuli is also disturbed (Grillon et al., 1990; Braff, 1993; Gray, 1995; Kapur, 2003). Functional neuroimaging studies recently confirmed that in at least some cerebral areas involved in the processing of salient stimuli, the hemodynamic response elicited by orienting to novel stimuli is greater in patients than in healthy participants (Laurens et al., 2005). Therefore, based on the orienting account for post-error slowing, increased rather than decreased slowing following unexpected outcomes can be predicted in patients when compared to controls. In the present study we investigated this prediction by using the same adaptive four-choice RT task designed to manipulate error rates as in previous studies (see Notebaert et al., 2009 and Núñez Castellar et al., 2010). Given that it has been shown that the slowing is strongly influenced by the error frequency, we leveled out performance in controls and patients by manipulating color discriminability. In this task we control the accuracy by making the color discrimination easier or harder depending on the subjects' performance. Participants performed the task in two conditions: 75\%-correct responses (expectancy for correct) and 35\%-correct responses (expectancy for error). We expect that patients with higher symptom severity will need more color (higher discriminability) than patients with low symptom severity. This prediction is made based on previous research showing that schizophrenic patients show sensory processing deficits (Slaghuis, 2004; Butler et al., 2005) and diminished performance when compared to controls in color vision tests (Shuwairi et al., 2002).

More important, however, on the basis of the orienting account we expect schizophrenic patients to show increased posterror slowing in the $75 \%$ correct condition and post-correct slowing in the $35 \%$ correct condition at short inter-trial intervals (ITIs). With longer ITIs, we expect that the effects of attention reorienting will be reduced. Remarkably, this prediction is opposing cognitive control theories stating that decreased ACC activity and decreased ERNs in schizophrenia should result in decreased post-error slowing.

\section{METHOD}

\section{PARTICIPANTS}

Eighteen healthy adults (eight males) and 18 patients with schizophrenia (14 males) participated in the experiment. All provided informed consent, had the approval of the local ethical committee, and participated in accord with the Declaration of Helsinki. Each had normal or corrected-to-normal vision. Four participants were excluded from analyses because they did not reach with the adaptive program the expected performance levels (performance more than 2, 5 standard deviations away from the group mean). Thus, data of 17 patients and 15 controls are here reported. The three excluded controls performed the task above the expected performance levels.

Controls were medication-free volunteers without a history of psychiatric or neurological illness. Patients were stable, partially remitted, medicated in- and outpatients recruited from St Norbertushuis Duffel psychiatric center. All patients met Diagnostic and Statistical Manual of Mental Disorders (4th ed.; DSM-IV; American Psychological Association, 1994) criteria for schizophrenia, as diagnosed by an institutional psychiatrist. Clinical status was characterized with the Positive and Negative Syndrome Scale (PANSS; Kay et al., 1987). All patients were mildly ill at the time of the testing (group mean PANSS total $65,2 \pm 22,4)$. Thirteen patients received stable doses of atypical antipsychotics as their primary medication, two received typical antipsychotics, and two typical antipsychotic adjunctive to the atypical medication. Patients and controls did not differ significantly in age and gender (see Table $\mathbf{1}$ ).

\section{TASK}

An adaptive four-choice RT task was used to manipulate error rates. This task was in principle equal to that published in previous studies (Notebaert et al., 2009; Núñez Castellar et al., 2010) but the response deadline and the amount of trials presented were modified. Stimuli were small colored squares presented centrally 
Table 1 | Demographic data by group and rating scale scores for patients.

\begin{tabular}{lllll}
\hline & $\begin{array}{l}\text { Healthy } \\
\text { controls }\end{array}$ & $\begin{array}{l}\text { Schizophrenia } \\
\text { patients }\end{array}$ & $\boldsymbol{t}$ & $\boldsymbol{p}$ \\
\hline Age & $32.1(8.1)$ & $33.1(6.5)$ & 0.41 & 0.68 \\
Sex & $6 \mathrm{M} / 9 \mathrm{~F}$ & $13 \mathrm{M} / 4 \mathrm{~F}$ & $-2.06^{*}$ & 0.09 \\
PANSS Positive & & $13.6 \pm 6.5$ & & \\
PANSS Negative & & $18.5 \pm 6.6$ & & \\
PANSS general & & $33.6 \pm 10.4$ & & \\
PANSS total & & $65.2 \pm 22.4$ & & \\
\hline
\end{tabular}

*Non-parametric Mann-Whitney comparison.

on a white background. The brightness of the colors was adjusted automatically in order to keep every participant's performance at a pre-specified level ( $35 \%$ or $75 \%$ accuracy). Colors are described according to the HSV color model with three parameters: hue $(0-360)$, saturation $(0-100)$, and value $(0-100)$. The four colors that were used in the practice trials were red $(20,100,80)$, yellow $(60,100,80)$, green $(120,100,80)$, and blue $(240,100,80)$. Participants responded to each of the four colors with one of the four buttons on a response box, using their left and right middle and index fingers. Four different color-to-button mappings were used, and participants were randomly assigned to one of these mappings.

Each trial started with a central fixation cross $(500 \mathrm{~ms})$ before the stimulus presentation. Then the stimulus was presented and remained on the screen for a maximum of $500 \mathrm{~ms}$ or until a response button was pressed. The response was immediately followed by feedback ("J" for correct and "F" for incorrect, corresponding to the Dutch words "juist" and "fout"). Following the feedback presentation four different ITIs were randomly intermixed ( $150 \mathrm{~ms}, 250 \mathrm{~ms}, 500 \mathrm{~ms}$, and $750 \mathrm{~ms})$. For the data analysis $150 \mathrm{~ms}, 250 \mathrm{~ms}$ were considered as short ITIs and $500 \mathrm{~ms}$ and $750 \mathrm{~ms}$ as long ITIs. The experiment started with a practice block (40 trials) without a response deadline. Later in a second practice block (40 trials) a response deadline of $2000 \mathrm{~ms}$ was introduced simultaneously with a feedback signal: "T", for "too slow" ("te laat," in Dutch). Afterwards two experimental blocks with the same response deadline were presented (200 trials). Each block corresponded to the $35 \%$ - and $75 \%$-accuracy rate manipulation. The order in which the blocks were presented was counterbalanced and within each block, participants received a short break. On every trial, the program calculated the accuracy of the last 20 trials and adjusted the color value by 1 value point when accuracy deviated from the specified level (75\% or $35 \%)$. The color value increased when accuracy was too low and decreased when accuracy was too high to reach the pre-specified accuracy levels (see more details in Notebaert et al., 2009). The experiment lasted about $30 \mathrm{~min}$.

\section{DATA ANALYSES}

We used the procedure described by Notebaert et al. (2009) for the data analysis: we excluded trials that occurred before a stable accuracy level was reached. Likewise, trials with RTs faster than $200 \mathrm{~ms}$ or slower than $2.000 \mathrm{~ms}$ (response deadline) and trials that were preceded by these trials were excluded. In total, $28.1 \%$ of the trials were excluded.

The results showed that the adaptation procedure worked as expected for the $75 \%$ and $35 \%$ correct conditions, with accuracies of $76 \%( \pm 4.2 \%)$ and $40 \%( \pm 3.1 \%)$ for the patients group, and of $75 \%( \pm 1.4 \%)$ and $38 \%( \pm 3.2)$ for the control group, respectively. A $t$-test showed that these accuracy rates did not differ between patients and controls in the $75 \%$ accuracy condition $[t(30)=1.35, p=0.18]$ or in the $35 \%$ accuracy condition $[t(30)=1.21, p=0.23]$. The order in which the conditions were administered did not yield significant effects; therefore, we omitted this factor in subsequent analyses.

\section{COLOR NEEDED TO REACH 35\% AND 75\% ACCURACY}

We used the adaptive program in order to obtain equal accuracy levels for patients and controls since we speculated that without this program, patients would make more errors, which would possibly lead to reduced post-error slowing (Notebaert et al., 2009). In order to stress the importance of this manipulation we calculated the amount of color needed (the value) for each participant. Although there was no overall group difference in color needed in both conditions [35: $t(30)=0.24, p=0.81 ; 75$ : $t(30)=0.61, p=0.54]$, the amount of color needed correlated significantly with PANSS scores for the patients (see Figure 1). In order to rule out the possibility that results were driven by outliers, outlier analyses were carried out. Outliers were identified by calculated the cook's distance measures for all the correlations reported. Cases with values above the percentile 50 (F-distribution) were identified as outliers. After excluding the cases labeled as outliers from the analyses, the significant results reported in the Figure 1 remained unchanged.

The significant correlation between the color needed for patients and the PANSS scores underlines the importance of the adaptive program since without the program patients with higher PANSS scores would have made more errors than patients with milder symptoms.

\section{POST-ERROR SLOWING}

Reaction times for correct and error trials in short and long ITIs for patients and controls in the current trial $(n)$, in the correct trials $(n+1)$ and the post-error slowing are reported in the Table 2.

For the analysis of the post-error slowing effect, RTs of correct trials were subjected to an analysis of variance (ANOVA) with Previous trial accuracy (correct vs. error), Accuracy condition (35\% correct vs. $75 \%$ correct) and ITI (short vs. long) as within-subjects factors and Group (control vs. patients) as between-subjects factor.

The results revealed a significant main effect of Group $[F(1,30)=14.38, p<0.001]$, indicating increased reaction times for patients when compared to controls $(857 \mathrm{~ms}$ vs. $700 \mathrm{~ms}$; $p<0.001)$. The main effects of Condition $[F(1,30)=2.84, p=$ $0.10]$, and Previous trial accuracy $[F(1,30)=3.7, p=0.06]$ were not significant. The main effect of ITI was also not significant $[F(1,30)=1.3, p=0.26]$.

The interaction between Condition and Accuracy of the previous trial was significant, $[F(1,14)=11.69, p<0.01]$, replicating 


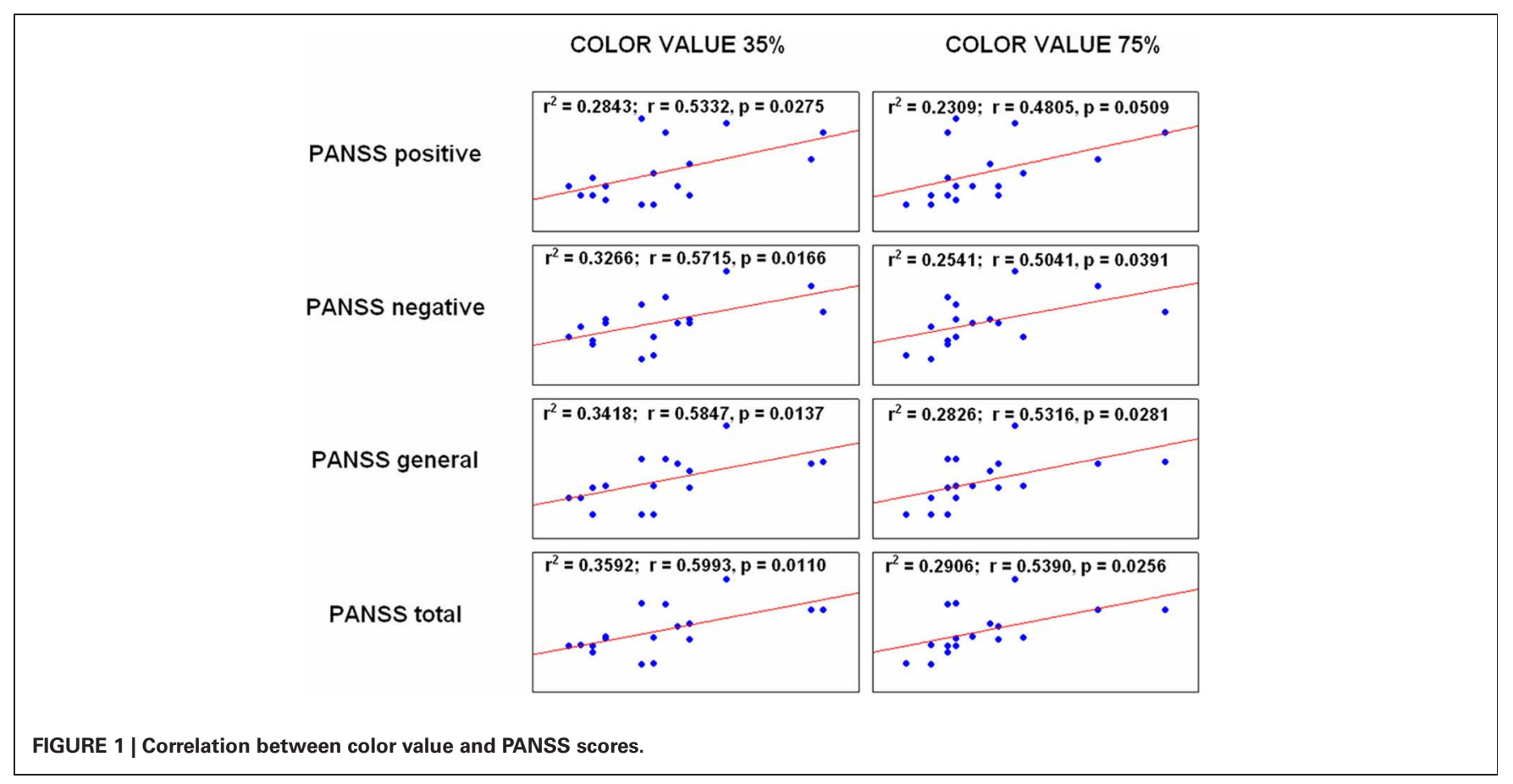

Table 2 | Reaction times for correct and error trials in short and long ITIs for patients and controls in the current trial ( $n$ ), in the correct trials $(n+1)$ and post-error slowing.

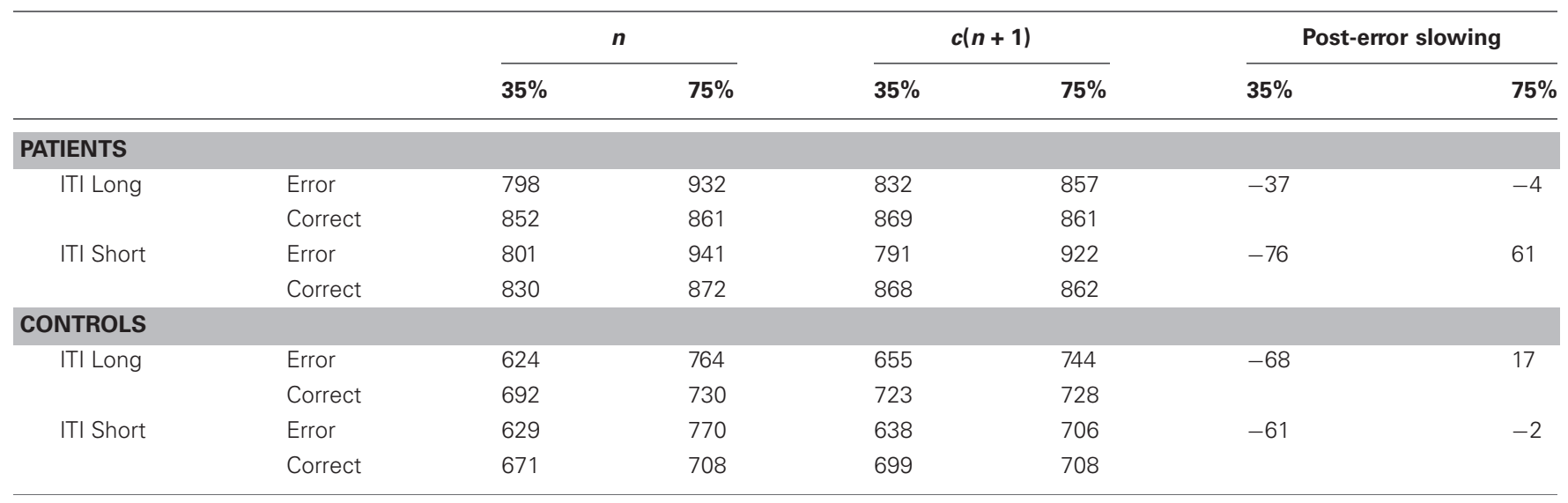

the study of Notebaert and colleagues (2009). The interaction between Condition, ITI, and Group was significant $[F(1,30)=$ 4.31, $p<0.05$ ], but more important for the interpretation of the results, the four way interaction between Condition, Previous accuracy, Group, and ITI was significant as well $[F(1,30)=$ 4.46, $p<0.05]$. Figure 2 shows that patients' slowing depends on the ITI while control participants' data are unaffected by ITI. ANOVAs performed separately for patients and control group confirm this pattern, showing that only for schizophrenic patients there is a significant interaction between Previous accuracy, Condition, and ITI $[F(1,16)=6.67, p<0.05]$ with increased slowing following unexpected outcomes in short ITIs, while for the control group this is not the case $[F(1,14)=$ $0.27, p=0.59]$. No other interactions were significant in the Four-Way ANOVA.

\section{DISCUSSION}

The aim of the present study was to further investigate errorrelated adjustments in schizophrenia. We tested the main prediction of a recent account that suggests the post-error slowing is driven by attentional mechanisms elicited by the unexpected nature of the error (Notebaert et al., 2009). For that purpose we used an adaptive four-choice reaction time task that allowed us to manipulate error rates. Every participant from the patient and control group performed a 35\% and $75 \%$ accuracy condition. Additionally the effect of short and long ITIs on the slowing was investigated.

Based on the orienting account we predicted that patients would show increased slowing at short ITIs considering their deficits in reorienting from the ongoing task and increased distraction by novel stimuli (Grillon et al., 1990; Braff, 1993). 


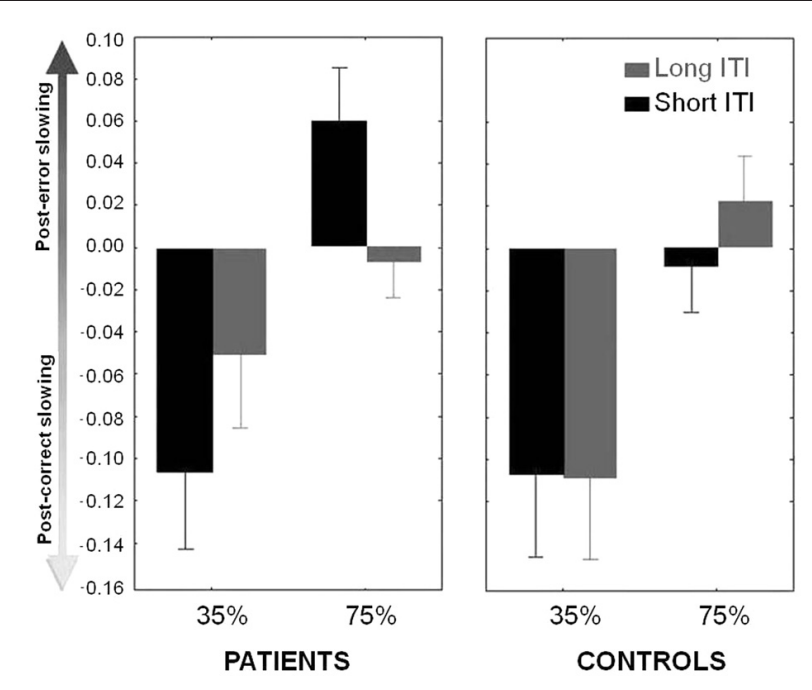

FIGURE 2 | Index of slowing (RTs after errors-RTs after correct responses/Individuals' mean RTs per condition). Positive difference values indicate post-error slowing while negative difference values indicate post-correct slowing. The graph shows increased slowing after unexpected correct and unexpected error responses in short ITIs for patients.

Consistent with the latter prediction, our results showed that in short ITIs, increased orienting to unexpected action outcomes in patients was observed. In other words, the size of the post-error and the post-correct slowing effects was significantly larger in patients than in controls in short ITIs. This finding is against one widely assumption of cognitive control theories, which suggest that the decreased ACC activity and decreased ERNs in schizophrenia that has consistently been reported in the extant literature (Kopp and Rist, 1994; Carter et al., 2001; Alain et al., 2002; Mathalon et al., 2002; Laurens et al., 2003; Bates et al., 2004; Kerns et al., 2005; Polli et al., 2008) should lead to decreased post-error slowing.

We speculate that since patients show deficits in performance monitoring as showed by studies demonstrating that the ERN amplitude is smaller in error trials at the same time that the ERN on correct trials is abnormally large (Kopp and Rist, 1999; Alain et al., 2002; Mathalon et al., 2002), it is likely that external feedback might be crucial for monitoring their performance. We hypothesize that attentional mechanisms might be more sensitive in patients than in controls to the external feedback producing a larger slowing. However, this is an issue that should be further investigated.

There is one aspect of the data that is unexpected, however. The control subjects do not show post-error slowing in both short and long ITIs in the 75\% accuracy condition, while they do show post-correct slowing in the $35 \%$ condition. The absence of posterror slowing has also been reported in another study (Bates et al., 2004) where matched controls were compared to schizophrenic patients but most of the studies observed post-error slowing in controls (Kopp and Rist, 1999; Mathalon et al., 2002; Polli et al., 2008). On the one hand an important methodological difference between the present study and previous studies that have reported post-error slowing in the $75 \%$ accuracy condition
(Notebaert et al., 2009; Núñez Castellar et al., 2010), is that the age-matched control subjects were on average 12 years older than the typical student population used in previous studies (e.g., Rabbitt and Rodgers, 1977; Jentzsch and Dudschig, 2009). Future studies should further investigate whether this is a factor that can influence the slowing in reaction times. On the other hand it is possible that in the $75 \%$ accuracy condition, errors are not surprising enough for the group of participants of the present study.

An important route for further research is to investigate error monitoring and behavioral adjustments following errors as a function of error type. Previous research has distinguished aware from unaware errors (e.g., Nieuwenhuis et al., 2001; Endrass et al., 2007), but one can also distinguish errors that were caused by perceptual factors (e.g., stimulus degradation), errors caused by short response deadlines forcing participants to give premature responses, errors caused by confusing mapping rules, errors caused by temporary confusion in a relatively simple mapping rule, errors caused by response conflict, and so on. One could argue that slowing after an error only makes sense when the error was caused by premature responding, as this would reduce the possibility of making yet another error. Consequently, within a framework where errors are considered to trigger adaptive behavior, one could explain the lack of post-error slowing in our control group by the fact that errors were not caused by premature responding. However, we like to add that in an identical experiment on a student population, where error RTs were not faster than correct RTs, we observed post-error slowing in this condition (Núñez Castellar et al., 2010). Nevertheless, we acknowledge that manipulating error type could be an important tool for increasing our understanding about error monitoring.

Two methodological aspects dissociate this study from previous ones (Kopp and Rist, 1999; Mathalon et al., 2002; Laurens et al., 2003; Polli et al., 2008). First, we leveled out performance in patients and matched their performance to controls. Overall accuracy levels play a key role in the modulation of post-error slowing in the sense that the more frequent the errors become, the smaller the size of the slowing gets (Notebaert et al., 2009). Importantly the correlation between average color needed for each patient and his or her PANSS scores for all subscales (see Figure 1) revealed that patients with higher PANSS would have made more errors when this was not controlled for. This is a relevant finding considering that in the present study it has been for the first time demonstrated that adaptive algorithms can successfully be used to control error rates in patients. This manipulation not only reduces drops-outs for a low number of error trials during data analysis, which is a common methodological problem faced by researchers investigating error-related processes, but also makes comparable the overall performance of patients with differentlevels of symptom severity. Consequently, we would like to plea for the use of similar adaptive programs when studying adaptive behavior in patients. Second, the manipulation of the ITIs showed to have an important effect in the slowing. When having a close look of the previous studies that have analyzed post-error slowing in schizophrenia, the ITIs fluctuated in a wide range going from 650 to $3000 \mathrm{~ms}$ (Bates et al., 2004; Laurens et al., 2005). Since here we have shown that short ITIs might enhance the size of the slowing, future studies should certainly consider this methodological aspect. 
Taken together, our results showed that patients with schizophrenia who show increased distraction by novel stimuli (Grillon et al., 1990; Braff, 1993; Gray, 1995; Kapur, 2003; Laurens et al., 2005) also showed increased slowing at short ITIs when compared to controls. This finding has important implications not only at the theoretical level providing important evidence for the idea that the post-error slowing could rely on different mechanisms that the ones involved in error monitoring but also for future clinical studies. One possible explanation for the pattern of results in the patients' group can be found in the orienting account of the post-error slowing, which suggests that the slowing does not necessarily rely on error monitoring but is rather modulated by attentional mechanisms. Researchers and clinicians are advised to be careful in interpreting this behavioral effect as a marker for cognitive control (Notebaert et al., 2009) and to take into account relevant methodological aspects, like the duration of the ITIs and the error rates, when drawing conclusions from this behavioral measure.

\section{ACKNOWLEDGMENTS}

This work was supported by a doctoral grant from the Ghent University special research fund (BOF 07/DOS/011).

\section{REFERENCES}

Alain, C., McNeely, H. E., He, Y., Christensen, B. K., and West, R. (2002). Neurophysiological evidence of error-monitoring deficits in patients with schizophrenia. Cereb. Cortex 12, 840-846.

American Psychological Association. (1994). Diagnostic and Statistical Manual of Mental Disorders, Fourth ed. Washington, DC: American Psychiatric Association.

Bates, A. T., Liddle, P. F., Kiehl, K. A., and Ngan, E. T. C. (2004). State dependent changes in error monitoring in schizophrenia. J. Psychiatr. Res. 38, 347-356.

Butler, P. D., Zemon, V., Schechter, I., Saperstein, A. M., Hoptman, M. J., Lim, K. O., Revheim, N., Silipo, G., and Javitt, D. C. (2005). Earlystage visual processing and cortical amplification deficits in schizophrenia. Arch. Gen. Psychiatry 62, 495-504.

Braff, D. L. (1993). Informationprocessing and attention dysfunctions in schizophrenia. Schizophr. Bull. 19, 233-259.

Carter, C. S., MacDonald, A. W., Ross, L. L., and Stenger, V. A. (2001). Anterior cingulate cortex activity and impaired self-monitoring of performance in patients with schizophrenia: an event-related fMRI study. Am. J. Psychiatry 158, 1423-1428.

Debener, S., Ullsperger, M., Siegel, M., Fiehler, K., von Cramon, D. Y., and Engel, A. K. (2005). Trial-by-trial coupling of concurrent electroencephalogram and functional magnetic resonance imaging identifies the dynamics of performance monitoring. J. Neurosci. 25, 11730-11737.

Endrass, T., Reuter, B., and Kathmann, N. (2007). ERP correlates of conscious error recognition: aware and unaware errors in an antisaccade task. Eur. J. Neurosci. 26, 1714-1720.

Friedman, D., Cycowicz, Y. M., and Gaeta, H. (2001). The novelty P3: an event-related brain potential (ERP) sign of the brain's evaluation of novelty. Neurosci. Biobehav. Rev. 25, 355-373.

Frith, C. D., and Done, D. J. (1989). Experiences of alien control in schizophrenia reflect a disorder in the central monitoring of action. Psychol. Med. 19, 359-363.

Gehring, W. J., Goss, B., Coles, M. G. H., Meyer, D. E., and Donchin, E. (1993). A neural system for error-detection and compensation. Psychol. Sci. 4, 385-390.

Gray, J. A. (1995). Dopamine release in the nucleus-accumbens - the perspective from aberrations of consciousness in schizophrenia. Neuropsychologia 33, 1143-1153.

Grillon, C., Courchesne, E., Ameli, R., Geyer, M. A., and Braff, D. L. (1990). Increased distractibility in schizophrenic-patients - electrophysiologic and behavioral evidence. Arch. Gen. Psychiatry 47, 171-179.

Hajcak, G., McDonald, N., and Simons, R. F. (2003). To err is autonomic: error-related brain potentials, ANS activity, and post-error compensatory behavior. Psychophysiology 40, 895-903.

Hajcak, G., and Simons, R. F. (2008). Oops!.. I did it again: an ERP and behavioral study of double-errors. Brain Cogn. 68, 15-21.

Jentzsch, I., and Dudschig, C. (2009). Why do we slow down after an error? Mechanisms underlying the effects of posterror slowing. Q. J. Exp. Psychol. 62, 209-218.

Johns, L. C., Rossell, S., Frith, C., Ahmad, F., Hemsley, D., Kuipers, E., and McGuire, P. K. (2001). Verbal self-monitoring and auditory verbal hallucinations in patients with schizophrenia. Psychol. Med. 31, 705-715.

Kapur, S. (2003). Psychosis as a state of aberrant salience: a framework linking biology, phenomenology, and pharmacology in schizophrenia. Am. J. Psychiatry 160, 13-23.
Kay, S. R., Fiszbein, A., and Opler, L. A. (1987). The positive and negative syndrome scale (PANSS) for schizophrenia. Schizophr. Bull. 13, 261-276.

Kerns, J. G., Cohen, J. D., MacDonald, A. W., Johnson, M. K., Stenger, V. A., Aizenstein, H., and Carter, C. S. (2005). Decreased conflictand error-related activity in the anterior cingulate cortex in subjects with schizophrenia. Am. J. Psychiatry 162, 1833-1839.

Kopp, B., and Rist, F. (1994). Error-correcting behavior in schizophrenic-patients. Schizophr. Res. 13, 11-22.

Kopp, B., and Rist, F. (1999). An eventrelated brain potential substrate of disturbed response monitoring in paranoid schizophrenic patients. $J$. Abnorm. Psychol. 108, 337-346.

Laming, D. R. J. (1968). Information Theory of Choice Reaction Times. New York, NY: Academic Press.

Laming, D. R. J. (1979). Autocorrelation of choice-reaction times. Acta Psychol. 43, 1381-1412.

Laurens, K. R., Kiehl, K. A., Ngan, E. T. C., and Liddle, P. F. (2005). Attention orienting dysfunction during salient novel stimulus processing in schizophrenia. Schizophr. Res. 75, 159-171.

Laurens, K. R., Ngan, E. T. C., Bates, A. T., Kiehl, K. A., and Liddle, P. F. (2003). Rostral anterior cingulate cortex dysfunction during error processing in schizophrenia. Brain 126, 610-622.

Leudar, I., Thomas, P., and Johnston, M. (1994). Self-monitoring in speech production - effects of verbal hallucinations and negative symptoms. Psychol. Med. 24, 749-761.

Malenka, R. C., Angel, R. W., Hampton, B., and Berger, P. A. (1982). Impaired central error-correcting behavior in schizophrenia. Arch. Gen. Psychiatry 39, 101-107.

Malenka, R. C., Angel, R. W., Thiemann, S., Weitz, C. J., and
Berger, P. A. (1986). Central error-correcting behavior in schizophrenia and depression. Biol. Psychiatry 21, 263-273.

Mathalon, D. H., Fedor, M., Faustman, W. O., Gray, M., Askari, N., and Ford, J. M. (2002). Responsemonitoring dysfunction in schizophrenia: an event-related brain potential study. J. Abnorm. Psychol. 111, 22-41.

Mlakar, J., Jensterle, J., and Frith, C. D. (1994). Central monitoring deficiency and schizophrenic symptoms. Psychol. Med. 24, 557-564.

Nieuwenhuis, S., Ridderinkhof, K. R., Blow, J., Band, G. P. H., and Kok, A. (2001). Error-related brain potentials are differentially related to awareness of response errors: evidence from an antisaccade task. Psychophysiology 38, 752-760.

Notebaert, W., Houtman, F., van Opstal, F., Gevers, W., Fias, W. and Verguts, T. (2009). Post-error slowing: an orienting account. Cognition 111, 275-279.

Núñez Castellar, E., Kühn, S., Fias, W., and Notebaert, W. (2010). Outcome expectancy and not accuracy determines posterror slowing: ERP support. Cogn. Affect. Behav. Neurosci. 10, 270-278.

Polli, F. E., Barton, J. J. S., Thakkar, K. N., Greve, D. N., Goff, D. C., Rauch, S. L., and Manoach, D. S. (2008). Reduced error-related activation in two anterior cingulate circuits is related to impaired performance in schizophrenia. Brain 131, 971-986.

Polli, F. E., Barton, J. J. S., Vangel, M., Goff, D. C., Iguchi, L., and Manoach, D. S. (2006). Schizophrenia patients show intact immediate error-related performance adjustments on an antisaccade task. Schizophr. Res. 82, 191-201.

Rabbitt, P. (1966). Errors and error correction in choice-response tasks. J. Exp. Psychol. Gen. 71, 264-272. 
Rabbitt, P., and Rodgers, B. (1977). What does a man do after he makes an error? An analysis of response programming. Q. J. Exp. Psychol. 29, 727-743.

Shuwairi, S. M., Cronin-Golomb, A., McCarley, R. W., and O'Donnell, B. F. (2002). Color discrimination in schizophrenia. Schizophr. Res. 55, 197-204.

Slaghuis, W. L. (2004). Spatio-temporal luminance contrast sensitivity and visual backward masking in schizophrenia. Exp. Brain Res. 156, 196-211.

Turken, U., Vuilleumier, P., Mathalon, D. H., Swick, D., and Ford, J. M (2003). Are impairments of action monitoring and executive control true dissociative dysfunctions in patients with schizophrenia? Am. J. Psychiat. 160, 1881-1883.

Conflict of Interest Statement: The authors declare that the research was conducted in the absence of any commercial or financial relationships that could be construed as a potential conflict of interest.

Received: 17 October 2011; paper pending published: 28 October 2011; accepted: 11 February 2012; published online: 29 February 2012.

Citation: Núñez Castellar E, Houtman F, Gevers W, Morrens M, Vermeylen S, Sabbe B and Notebaert W (2012) Increased orienting to unexpected action outcomes in schizophrenia. Front. Hum. Neurosci. 6:32. doi: 10.3389/fnhum. 2012.00032

Copyright (c) 2012 Núñez Castellar, Houtman, Gevers, Morrens, Vermeylen, Sabbe and Notebaert. This is an openaccess article distributed under the terms of the Creative Commons Attribution Non Commercial License, which permits non-commercial use, distribution, and reproduction in other forums, provided the original authors and source are credited. 